\title{
Measurement of subpicosecond bunch lengths using coherent Smith-Purcell radiation
}

\author{
S.E. Korbly, A. S. Kesar, and R. J. Temkin \\ Plasma Science and Fusion Center, Massachusetts Institute of Technology, Cambridge, Massachusetts 02139, USA
}

J. H. Brownell

Department of Physics and Astronomy, Dartmouth College, Hanover, New Hampshire 03755, USA

(Received 4 May 2005; published 24 February 2006)

\begin{abstract}
We report the use of coherent Smith-Purcell radiation to measure the bunch length of femtosecondscale, $15 \mathrm{MeV}$ electron bunches produced by a $17 \mathrm{GHz}$ rf accelerator. The Smith-Purcell radiation was produced by passing a train of electron bunches above a metal grating. The radiation was verified as Smith-Purcell radiation by measuring the resonance condition, dependence on beam current, and dependence on beam height above the grating. Measurements of the intensity of the radiation vs emission angle were analyzed to obtain the bunch length. The accelerator was operated in two different modes, producing bunches that were determined to have bunch lengths of 600 and $1000 \pm 200 \mathrm{fs}$. These nondestructive bunch length measurements were found to agree well with an independent, but destructive, measurement using a microwave deflecting cavity.
\end{abstract}

DOI: 10.1103/PhysRevSTAB.9.022802

PACS numbers: 41.60.- m, 29.27.Fh, 41.75.Ht, 52.70.Kz

\section{INTRODUCTION}

The measurement and diagnosis of ultrashort, i.e., subpicosecond, electron bunches is important for linear colliders, free electron lasers (FELs) and plasma accelerators. Various different techniques have demonstrated the measurement of subpicosecond bunch lengths including streak cameras [1], rf deflecting structures [2,3], electro-optic techniques [4], coherent synchrotron radiation [5,6], and coherent diffraction radiation $[7,8]$. The ideal method will be nondestructive, have sub 100 fs resolution and be extendable to very short bunches, i.e., tens of femtoseconds. One of the most promising bunch length measurement techniques, which has minimal effect on the beam, is Smith-Purcell radiation (SPR) $[9,10]$. SPR, which occurs when a charged particle passes over a periodic structure, was first observed in 1953 [11]. SPR has several advantages over other coherent radiation generation techniques such as synchrotron, transition and diffraction radiation. First, the intensity of radiation is higher by the number of grating periods, $N_{g}$, which is typically on the order of 10 to 100. Additionally, the radiation is angularly dispersed in frequency according to the SPR dispersion relation

$$
\lambda=\frac{l}{n}\left(\frac{1}{\beta}-\cos \theta\right)
$$

where $\lambda$ is the wavelength of the radiation, $n$ is the order of the radiation, $l$ is the grating period, $\beta$ is the ratio of the electron velocity to the speed of light, and $\theta$ is the observation angle as shown in Fig. 1. The first demonstration of a SPR bunch length measurement was performed for $14 \mathrm{ps}$ bunches $[12,13]$. The experimental setup described in this paper was previously used to study frequency locking of SPR $[14,15]$. This manuscript expands upon the previous bunch length research, reporting additional data and ex- perimental details and demonstrating the first subpicosecond bunch length measurement using SPR.

\section{THEORY}

For one electron the radiated energy density per unit frequency of the $n$th order of SPR can be written as [16]

$$
\frac{d I_{n}}{d \omega}=\frac{e^{2} N_{g} l}{8 \pi^{2} \epsilon_{o} c^{2}} \omega \sin \theta \int\left|R_{n}\right|^{2} \exp \left[-\frac{2 x_{o}}{\lambda_{e}}\right] \sin ^{2}(\phi) d \phi,
$$

where $x_{o}$ is the height of the electron above the grating, $\phi$ is the azimuthal angle with respect to the $x y$ plane, $\left|R_{n}\right|^{2}$ is a grating efficiency factor which is a function of the grating profile, wavelength and order of radiation [17], and $\lambda_{e}$ is the "evanescent wavelength" defined as

$$
\lambda_{e}=\lambda \frac{\beta \gamma}{2 \pi \sqrt{1+\beta^{2} \gamma^{2} \sin ^{2} \theta \sin ^{2} \phi}},
$$

where $\gamma$ the Lorentz factor $\left(1-\beta^{2}\right)^{-1 / 2}$.

For $N_{e}$ electrons in a bunch, coherent radiation is produced for wavelengths longer than the bunch length $[18,19]$. The total radiated intensity becomes

$$
\left(\frac{d I_{n}}{d \omega}\right)_{N_{e}}=\left(\frac{d I_{n}}{d \omega}\right)\left(N_{e} S_{\mathrm{inc}}+N_{e}^{2} S_{\mathrm{coh}}(\omega)\right)
$$

where $\left(d I_{n} / d \omega\right)$ is given by Eq. (2) while setting $x_{o}=0$. Consider the electron bunch distribution functions to be three independent functions, one for each dimension, $X(x)$, $Y(y), Z(z)$. The incoherent form factor becomes

$$
S_{\text {inc }}=\int_{h}^{\infty} d x X(x) e^{-2 x / \lambda_{e}}
$$

where $h$ is the maximum height of the grating. The coher- 


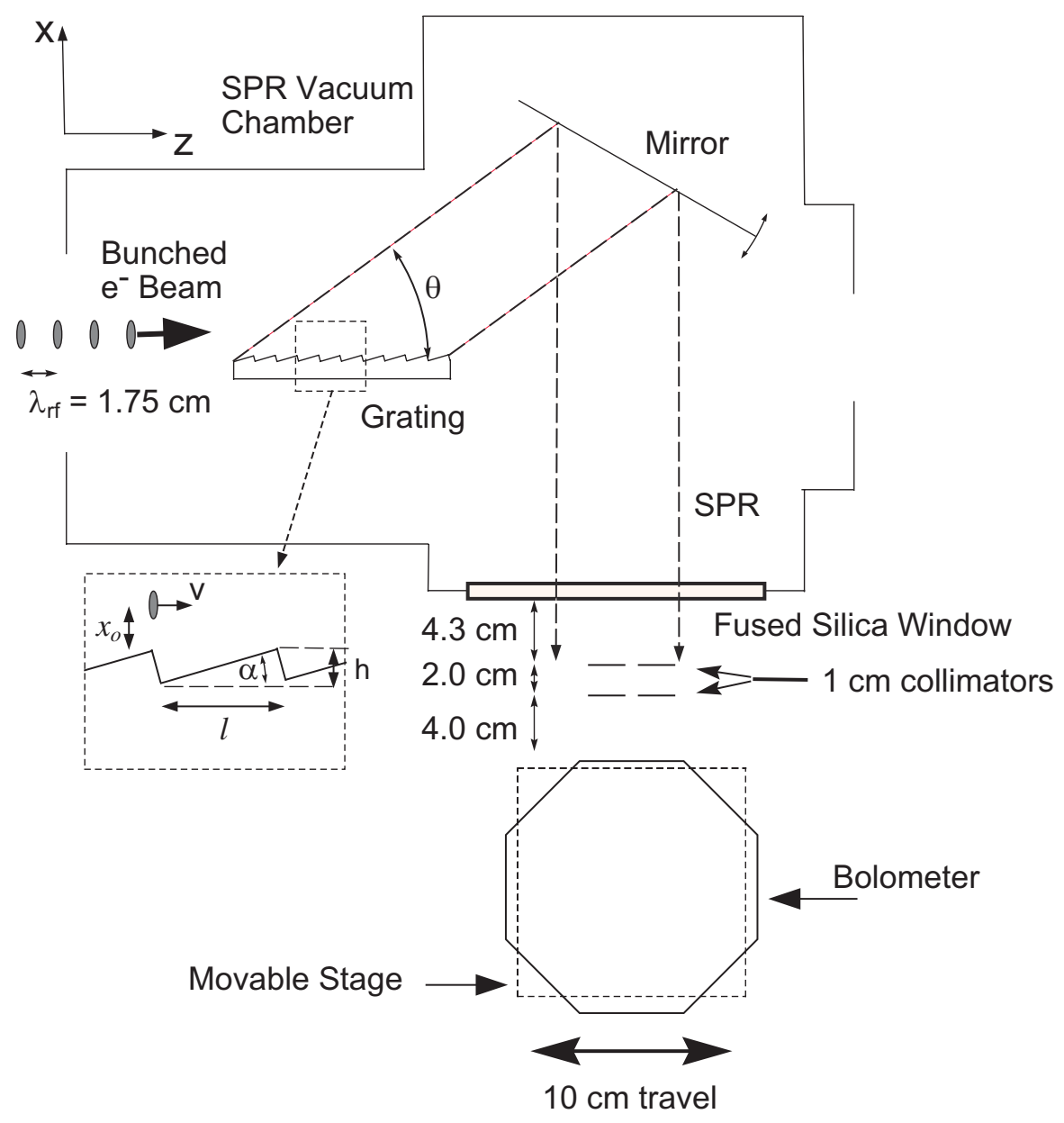

FIG. 1. SPR experimental setup. The train of bunched electrons is traveling along the $z$ direction above a metallic echelle grating. The diffracted radiation is measured by a detector located outside of the vacuum chamber.

ent form factor is

$$
S_{\mathrm{coh}}=\left|\int_{h}^{\infty} d x X(x) e^{-x / \lambda_{e}} \tilde{Y}\left(k_{y}\right) \tilde{Z}(\omega)\right|^{2}
$$

where $\tilde{Y}$ and $\tilde{Z}$ are the Fourier transforms of the bunch distribution functions. The main contribution to the coherence term, Eq. (6), comes from the longitudinal distribution, $\widetilde{Z}(\omega)$, which decays exponentially for wavelengths shorter than the bunch length.

Two 3D codes were utilized to predict and analyze the SPR. An image charge code based upon [20] was used to design the experiment. The experiment was designed by optimizing the blaze angle and periodicity to provide maximum radiation intensity and maximum angular sensitivity with respect to the mirror, window size and beam parameters according to the formulation in [20]. An electric field integral equation code which follows [21] and considers finite grating effects was used to analyze the data.

\section{EXPERIMENTAL SETUP}

The electron beam is produced by a linac, built by Haimson Research Corporation (HRC), which consists of a $515 \mathrm{kV}$ electron gun, a chopper-prebuncher and three lens injector and a quasiconstant gradient accelerating structure consisting of 94 cavities that operate in the $2 \pi / 3$ mode [22]. The linac is powered by the HRC relativistic klystron $[23,24]$ which was typically operated at $15 \mathrm{MW}$ of rf power at $17.140 \mathrm{GHz}$ in $\sim 100 \mathrm{~ns}$ pulses (flat top). The electron beam was approximately $1 \mathrm{~mm}$ in diameter (FWHM) with an emittance of $3 \pi \mathrm{mm}$-mrad. The beam current was $150 \mathrm{~mA}$, corresponding to $5.6 \times 10^{7}$ electrons per bunch, and the beam centroid height above the grating was set to $0.75 \mathrm{~mm}$, except for the specific cases where dependence on beam current and height were measured.

The chopper-prebuncher injection system was designed to produce a high quality beam at the entrance to the linac [25]. The chopper cavity is designed to produce bunches as short as $100^{\circ}$ using a peak rf input power of $1.4 \mathrm{~kW}$. The prebuncher was designed to compress these bunches down to $15^{\circ}$ using a peak power of $1.5 \mathrm{~kW}$. The $15^{\circ}$ input bunch is further compressed during acceleration in the linac down 
to about $1^{\circ}$, which corresponds to $160 \mathrm{fs}$ or $50 \mu \mathrm{m}$ at 17.14 GHz. Independently controlled phase shifters and power attenuators are used to adjust the phase relationship between the injector cavities and the linac and the power levels of each cavity. Adjustment of the phase and power levels of the chopper and prebuncher cavities allows for a range of bunch lengths, $1^{\circ}-10^{\circ}$, to be produced. When the accelerator produces $1^{\circ}$ bunches the charge per bunch is small. All runs described in this paper have $>500 \mathrm{fs}(\sim$ $3^{\circ}$ ) bunch length.

The SPR vacuum chamber shown in Fig. 1 houses the grating, a mirror and a window and is located $3 \mathrm{~m}$ downstream from the exit of the linac. A toroidal focusing lens is located $2 \mathrm{~m}$ upstream from the grating, allowing the beam to be focused to an emittance limited spot size of $1.0 \mathrm{~mm}$. Two echelle type gratings were used with periods of 10 and $6 \mathrm{~mm}$. Both gratings had an overall length of $100 \mathrm{~mm}$ and a blaze angle of $10^{\circ}$. The gratings were built of OFHC copper and the grating parameters were chosen to optimize the SPR for a 1.0 and $0.5 \mathrm{ps}$ bunch, respectively, using the formulation described in [20]. The grating height can be adjusted with two remote controlled stepper motor feedthroughs allowing both the front and the back of the grating to be raised or lowered independently.

The radiation is directed out of the vacuum chamber via a flat copper mirror of overall length of $12.7 \mathrm{~cm}$ and passes through a $10 \mathrm{~cm}$ diameter fused silica window. The mirror angle is adjusted via a remote controlled stepper motor. The absorption of fused silica at radiation frequencies $(100-800 \mathrm{GHz})$ is accounted for by using published data [26-29]. The absorption due to air [30] was considered negligible $(0.2 \mathrm{~dB})$ over the $20 \mathrm{~cm}$ distance that the radiation traveled to reach the detector.

The SPR was measured with an Infrared Laboratories Si bolometer which was cooled to liquid helium temperatures. The detector had a measured responsivity of $2 \times$ $10^{4} \mathrm{~V} / \mathrm{W}$. Two $1 \mathrm{~cm}$ collimators were placed between the window and the detector to reject stray radiation and to reduce the angular acceptance. The angular acceptance of the detector can be approximated as the radius of the collimator divided by the distance from the collimator to the detector element $\left(\sim 0.5 / 10 \simeq 3^{\circ}\right)$.

The detector was mounted on a translation stage which allowed it to be moved parallel to the window. The angular distribution was measured by moving the detector across the window in $1 \mathrm{~cm}$ steps, corresponding to the collimator width, for each mirror position. The intensity for each emission angle was then computed by summing the measured intensity for each detector position to give the total emitted radiation at a particular angle. The detector and associated collimators are shown in Fig. 1.

\section{RESULTS}

The Smith-Purcell resonance condition was verified with three band pass filters $(183,400$, and $800 \mathrm{GHz})$ while

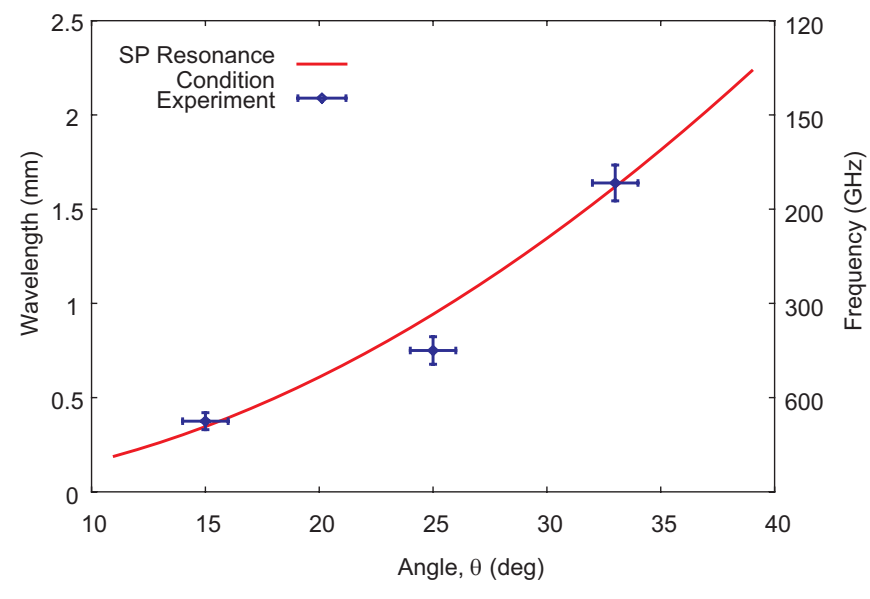

FIG. 2. (Color) Plot of the Smith-Purcell resonance condition and the measured experimental data for the $10 \mathrm{~mm}$ grating.

changing the collection angle. A plot of the resonance condition and the three frequencies for the $10 \mathrm{~mm}$ grating is shown in Fig. 2. The dependence of the SPR intensity on beam current was measured and is shown in Fig. 3. From Eq. (4) the radiated energy scales as

$$
\left(\frac{d I_{n}}{d \omega}\right)_{N_{e}} \propto N_{e}^{2} \propto I^{2}
$$

for coherent radiation. The measured dependence on beam current agrees well with the theoretical quadratic scaling. The detector and mirror were held fixed while the beam current was varied by changing the second focusing lens in the injector. The beam parameters: energy, transverse size and bunch length are constant until beam loading effects start to significantly affect the electron bunch acceleration ( $>200 \mathrm{~mA}$ ). The dependence of the SPR on the height of the beam was measured by adjusting the beam height in steps of $0.5 \mathrm{~mm}$. The measured intensity is shown in Fig. 4 along with the least squares fit. The mirror was held fixed at an observation angle of $\theta=23.5^{\circ}$. The beam energy was

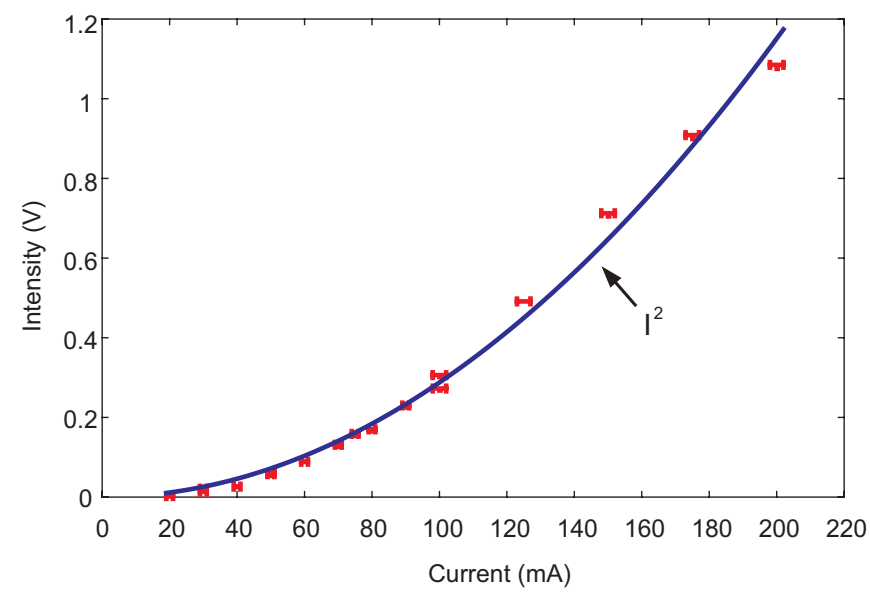

FIG. 3. (Color) Plot of the measured SPR intensity as a function of beam current. A curve proportional to the square of the beam current, I, is also shown. 


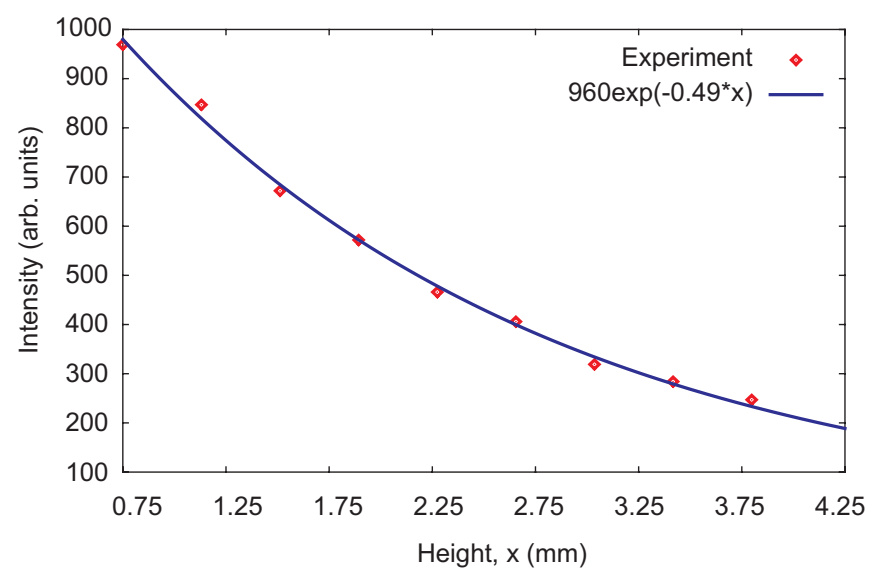

FIG. 4. (Color) Plot of the measured SPR intensity as a function of beam height above the grating.

$15.5 \mathrm{MeV}(\gamma=31)$ and the beam current was $150 \mathrm{~mA}$. The dependence of SPR on height above the grating is described in Eq. (6) which can be approximated as having a height dependence $\exp (-4 \pi x / \beta \gamma \lambda)$ when $\sigma_{x} \ll \lambda_{e}$. For this experiment $\sigma_{x} \sim 1 \mathrm{~mm}$ and $\lambda_{e} \sim 4 \mathrm{~mm}$ using the known values of $\beta \gamma \lambda$. The predicted height dependence is then $\exp (-0.489 x)$, where the bunch height above the grating, $x$, is in millimeters. The least squares fit to the data of Fig. 4 gives an exponent with a constant of $0.49 \pm 0.01$. The agreement is very good.

Measurements of the angular distribution of the radiation were made with the bolometer under two different accelerator operating modes. In the first measurement the accelerator was run in "long" bunch mode where $0.4 \mathrm{~kW}$ of rf power was supplied to the prebuncher and no power to the chopper. For the second measurement the accelerator

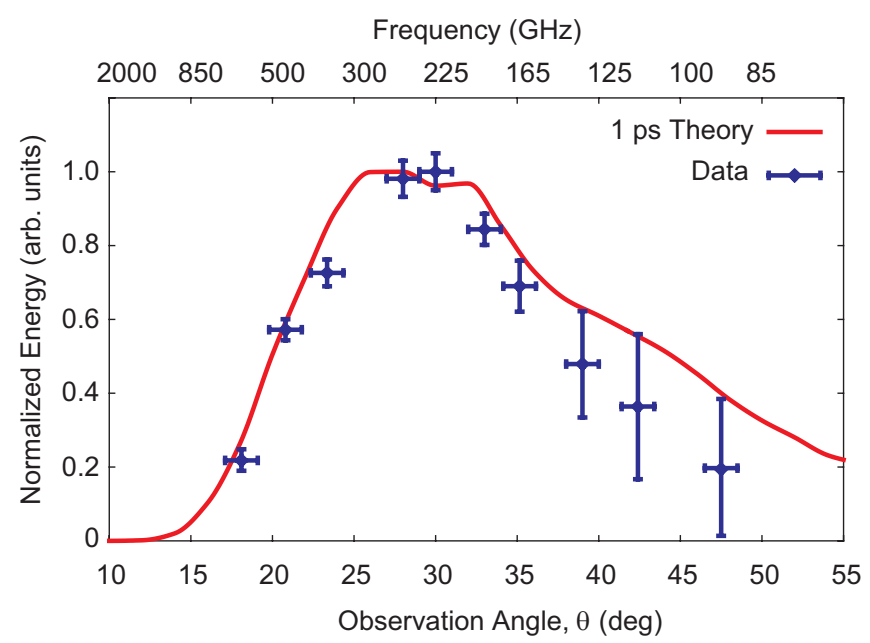

FIG. 5. (Color) Plot of the angular distribution of SPR normalized energy from the $10 \mathrm{~mm}$ grating as measured by the $\mathrm{Si}$ bolometer. The theoretical curve is shown for a $15 \mathrm{MeV}$ beam with a 1.0 ps FWHM Gaussian longitudinal distribution, and $1 \mathrm{~mm}$ FWHM Gaussian transverse distribution. First published in [15].

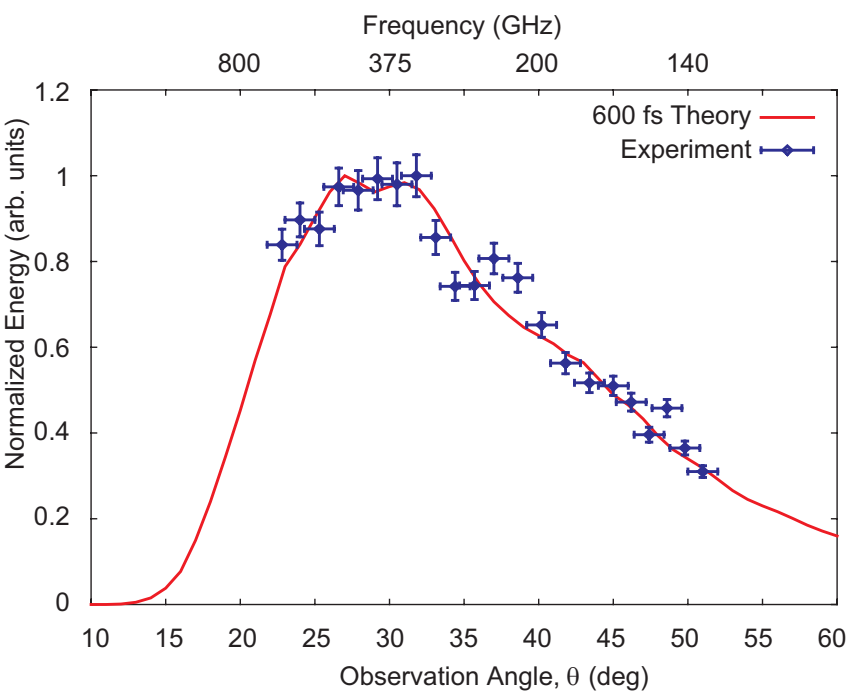

FIG. 6. (Color) Plot of the angular distribution of SPR normalized energy from the $6 \mathrm{~mm}$ grating as measured by the $\mathrm{Si}$ bolometer. The theoretical curve is shown for a $15 \mathrm{MeV}$ beam with a 600 fs FWHM Gaussian longitudinal distribution, and $1 \mathrm{~mm}$ FWHM Gaussian transverse distribution.

was run in "short" bunch mode with $1.2 \mathrm{~kW}$ of power supplied to the prebuncher and $1.1 \mathrm{~kW}$ of power to the chopper. Two measurements of angular distribution intensity are shown in Figs. 5 and 6 . The theoretical angular distribution of SPR for a $15 \mathrm{MeV}$ beam with transverse distribution of $1 \mathrm{~mm}$ FWHM and Gaussian longitudinal distributions of 1.0 ps FWHM and 600 fs FWHM, respectively, are also plotted. Since the form factor, defined in Eq. (6), falls off exponentially when the wavelength is shorter than the bunch length the fit is most sensitive to the high frequency data. Figure 7 shows the 600 fs data

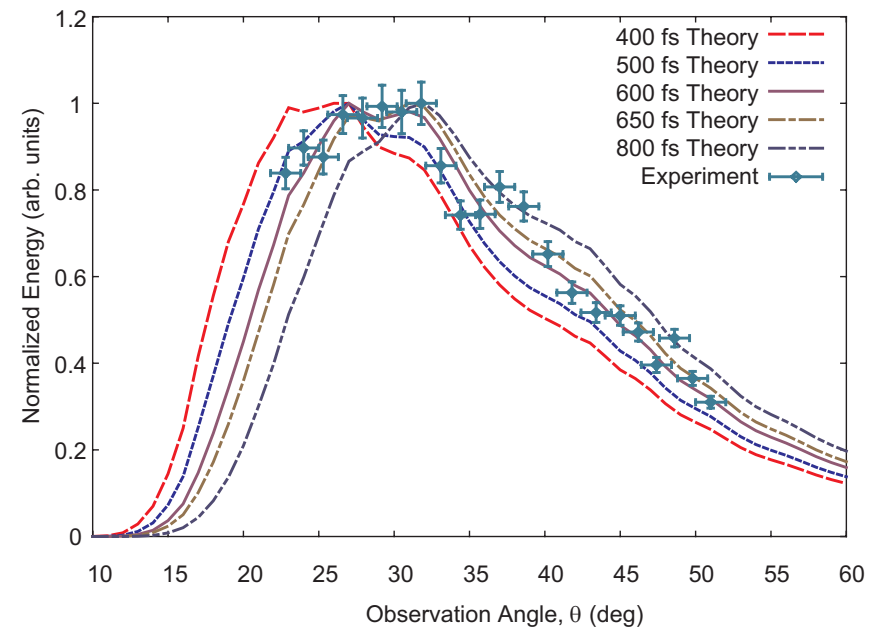

FIG. 7. (Color) Plot of the angular distribution of SPR normalized energy from the $6 \mathrm{~mm}$ grating as measured by the $\mathrm{Si}$ bolometer. The theoretical curve is shown for a $15 \mathrm{MeV}$ beam with various Gaussian longitudinal distributions, and $1 \mathrm{~mm}$ FWHM Gaussian transverse distribution. 
with theoretical distributions for several different longitudinal bunch lengths. Figure 7 is used to estimate that the bunch length uncertainty by this fitting is \pm 0.2 ps.

\section{CONCLUSION}

SPR from $15 \mathrm{MeV}$ electron bunches was measured. The SPR resonance condition was verified using several band pass filters. Additionally, the dependence of the radiation on current and beam height were measured and agree very well with theory. The measured radiated energy, $\sim 50 \mathrm{~nJ}$, is within an order of magnitude of theoretical predictions [20,21]. The measurement of the angular distribution of the SPR determined the longitudinal bunch length to be 1.0 $\pm 0.2 \mathrm{ps}$ when operating in long bunch mode and $600 \pm$ $200 \mathrm{fs}$ in short bunch mode. In Fig. 5 the larger error bars at lower frequency are due to the divergence of the SPR and the lower collection efficiency of the collimation system at the corresponding angles. In Fig. 6 measurement of the high frequency rolloff is restricted due to geometrical constraints of the grating, mirror and the vacuum chamber. Figure 7 shows that the best fit of the experimental data is greater than $400 \mathrm{fs}$ and less than $800 \mathrm{fs}$ and demonstrates the capability of measuring subpicosecond electron bunch lengths with SPR. The bunch length measurements using SPR were verified using an independent circularly polarized deflection measurement [3].

The use of SPR as a bunch length diagnostic has the advantage that it is a nondestructive technique and is important for FEL's, next generation linear colliders and plasma accelerators. Unlike other techniques such as the electro-optic and streak camera techniques, SPR has no inherent limitations on the minimum bunch that can be measured. Several other advantages might include lower cost and complexity and the possibility of performing a single shot bunch length monitor by using an array of detectors.

\section{ACKNOWLEDGMENTS}

This work was supported by the Department of Energy, Division of High Energy Physics, Contract No. DE-FG0291ER40648. The authors would like to thank Dr. Jake Haimson and Haimson Research Corporation for their help and advice in running the accelerator, Ivan Mastovsky, Roark Marsh, and Dr. Michael Shapiro for their help in running the experiments.

[1] T. Watanabe et al., Nucl. Instrum. Methods Phys. Res., Sect. A 480, 315 (2002).

[2] D. X. Wang, G. A. Krafft, and C. K. Sinclair, Phys. Rev. E 57, 2283 (1998).
[3] J. Haimson, in Advanced Accelerator Concepts: Eleventh Advanced Accelerator Concepts Workshop, AIP Conf. Proc. No. 737 (AIP, New York, 2004), pp. 95-108.

[4] G. Berden, S. P. Jamison, A. M. MacLeod, W. A. Gillespie, B. Redlich, and A. F. G. van der Meer, Phys. Rev. Lett. 93, 114802 (2004).

[5] K. Ishi et al., Phys. Rev. A 43, 5597 (1991).

[6] D. X. Wang et al., Appl. Phys. Lett. 70, 529 (1997).

[7] O. Haeberle, P. Rullhusen, J. M. Salome, and N. Maene, Phys. Rev. E 52, 6787 (1995).

[8] A. H. Lumpkin et al., Nucl. Instrum. Methods Phys. Res., Sect. A 475, 470 (2001).

[9] D. C. Nguyen, Nucl. Instrum. Methods Phys. Res., Sect. A, 393, 514 (1997).

[10] M. C. Lampel, Nucl. Instrum. Methods Phys. Res., Sect. A 385, 19 (1997).

[11] S. Smith and E. Purcell, Phys. Rev. 92, 1069 (1953).

[12] A. Doria et al., Nucl. Instrum. Methods Phys. Res., Sect. A 483, 263 (2002).

[13] G. Doucas et al., Phys. Rev. ST Accel. Beams 5, 072802 (2002).

[14] S.E. Korbly et al., in Proceedings of the Particle Accelerator Conference, Portland, OR, 2003 (IEEE, Piscataway, NJ, 2003), pp. 2536.

[15] S. E. Korbly, A. S. Kesar, J. R. Sirigiri, and R. J. Temkin, Phys. Rev. Lett. 94, 054803 (2005).

[16] P. M. van den Berg, J. Opt. Soc. Am. 63, 1588 (1973).

[17] O. Haeberle, P. Rullhusen, J. M. Salome, and N. Maene, Phys. Rev. E 49, 3340 (1994).

[18] S. Nodvick and D. S. Saxon, Phys. Rev. 96, 180 (1954).

[19] C. J. Hirschmugl, M. Sagurton, and G. P. Williams, Phys. Rev. A 44, 1316 (1991).

[20] J. H. Brownell, J. Walsh, and G. Doucas, Phys. Rev. E 57, 1075 (1998).

[21] A. S. Kesar, Phys. Rev. ST Accel. Beams 8, 072801 (2005).

[22] J. Haimson and B. Mecklenburg, in Proceedings of the Particle Accelerator Conference, Dallas, TX, 1995 (IEEE, Piscataway, NJ, 1995), pp. 755.

[23] J. Haimson et al., in Pulsed RF Sources for Linear Colliders, AIP Conf. Proc. No. 337 (AIP, New York, 1994), pp. 146.

[24] J. Haimson and B. Mecklenburg, in High Energy Density and High Power RF: Proceedings of the 6th Workshop on High Energy Density and High Power RF, AIP Conf. Proc. No. 691 (AIP, New York, 2003), pp. 34-35.

[25] J. Haimson and B. Mecklenburg, in Proceedings of the Particle Accelerator Conference, San Francisco, CA, 1991 (IEEE, Piscataway, NJ, 1991), pp. 3183-3185.

[26] K. K. Mon and A. J. Sievers, Appl. Opt. 14, 1054 (1975).

[27] T. J. Parker et al., Infrared Phys. 18, 215 (1978).

[28] I. H. Hutchinson, Infrared Phys. 22, 117 (1982).

[29] D. Grischkowsky et al., J. Opt. Soc. Am. B 7, 2006 (1990).

[30] N. G. Yaroslavsky and A. E. Stanevich, Appl. Opt. 7, 380 (1959). 